\title{
PREMIUM CALCULATION FROM TOP DOWN
}

\author{
By HaNS BÜHLMANN* \\ Zürich
}

\section{SUMMARY}

This paper is intended to show how premiums are related to the stability criterion imposed on a portfolio of risks and to the dividend requirements for the capital invested into the insurance operation. The point is that premium calculation should be seen as a consequence of the strategic concepts adopted by the insurance carrier.

\section{INSURANCE AS A DYNAMIC SYSTEM}

All insurance activities can be viewed as an input-output system, namely as follows

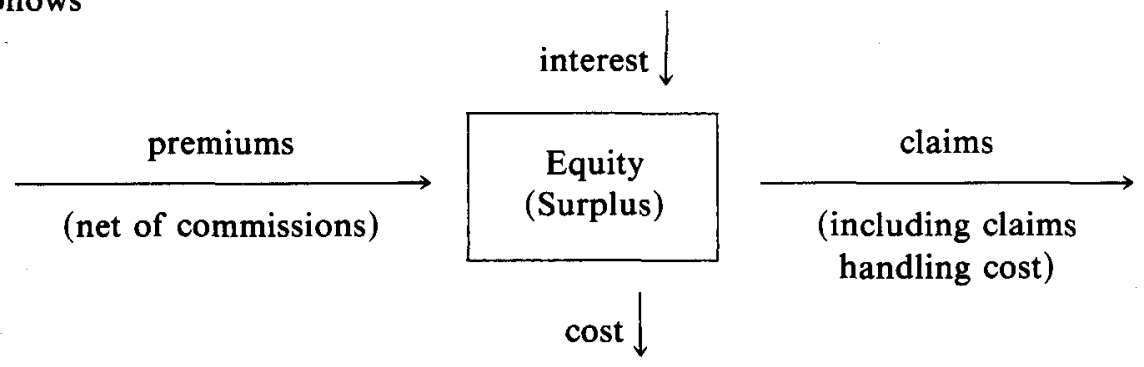

To fix the ideas think of yearly insurance contracts only and let

$P_{t} \sim$ premiums (net of commissions) in year $t$,

$S_{t} \sim$ claims incurred, i.e., paid and reserved (including claims handling costs) in year $t$,

$C_{t} \sim$ all costs incurred in year $t$ (except those already considered in $P_{t}$ and $S_{t}$ ),

$i_{t} \sim$ interest rate for year $t$,

$R_{t} \sim$ equity (surplus) at end of year $t$.

For simplicity assume that

premiums, claims, interest and cost are paid at the end of each year. More realistically you can think of these quantities (premiums, claims, costs) as present values per end of the risk year to which they "belong". Assume then that these present values are known with certainty.

OBSERVE. By this assumption we have eliminated reserving problems (=problems inherent in the uncertainty of the present values just mentioned). This is

* Lecture delivered at the Macquarie Seminar during the ICA 1984 in Sydney. 
obviously only done to concentrate on the pure premium calculation problems in this paper.

Then the basic relation is

$$
R_{t}=\left(1+i_{t}\right) R_{t-1}+P_{t}-C_{t}-S_{t}, \quad t=1,2,3, \ldots
$$

$\boldsymbol{R}_{0}$ stands for the initial equity, or the operative capital which is initially "invested" into the insurance operation. Observe also that from the dynamic point of view only the difference premiums minus costs (=net premium $\Pi_{t}$ ) is relevant. Hence we rewrite our basic relation as

$$
R_{t}=\left(1+i_{t}\right) R_{t-1}+\Pi_{t}-S_{t}, \quad t=1,2,3, \ldots
$$

\section{STABLE FUNCTIONING OF INSURANCE}

It is important to note that our Dynamic System's Approach makes only sense for a portfolio of risks (or even better: for the total or risks assumed by an insurance company).

Hence (1) is a relation between sums of net premiums $\Pi_{t}$ and sums of claims $S_{t}$. Insurance is stable if $\Pi_{t}$ and $S_{t}$ are in equilibrium with each other. We express this stable functioning by the postulate

$$
R_{t} \geqslant 0 \text { for all } t=1,2,3, \ldots
$$

It is important to note that (2) expresses our goal that net premiums and claims should not differ too widely. As a matter of fact (2) requires that the initial operative capital $R_{0}$ should be sufficient for the whole insurance operation over time.

Of course equilibrium could be formulated in other ways. The one chosen here-postulating that no additional capital should be needed during the insurance operation-seems reasonable in a portfolio without growth. We shall come back to the case of growth in a later paragraph.

\section{FORMS OF INSURANCE}

\subsection{Insurance Pools}

Assume that claims $S_{t}$ and costs $C_{t}$ are produced by a process which is outside of the insurance company's control. If the insurance scheme is such that one can compensate expenditures (claims $S_{t}$ and costs $C_{t}$ ) immediately and fully by the premiums $P_{t}$ to be received one can easily achieve equilibrium without any capital. Such an insurance arrangement is called a pool. It is characterised by the relations

$$
\left.\begin{array}{rl}
R_{t} & =0, \\
\Pi_{t} & =S_{t}, \\
\left(P_{t}\right. & \left.=S_{t}+C_{t}\right),
\end{array}\right\} \quad t=0,1,2, \ldots
$$

Such pools do exist in practice. 
Their advantage: no need of capital

Their disadvantage: premiums are only known a posteriori. Insurance effect is only achieved if the individual risk does not share in the total net premium in proportion to its claims, which creates "solidarity payments" between the members of the pool.

\subsection{Prospectively Calculated Premiums}

Except in cases where the pooling arrangement is an acceptable form of insurance to all pool members, one usually wants to transact insurance as insurance business. This means that before inception of the risk one wants to know the extent of the insurance cover on one side, the premium to be paid for the cover on the other side.

Hence arises the need to calculate $P_{t}$ in advance. I shall do this in the simple case (for didactical reasons) where the evolution of the portfolio is stationary and where interests on equity are absorbed by costs and/or by claims.

Therefore let me assume

$$
\begin{aligned}
& \left.\begin{array}{l}
i_{t}=0 \\
P_{t}=P \\
C_{t}=C
\end{array}\right\} \quad \text { for all } t \\
& S_{t} \quad(t=1,2, \ldots) \text { independent and identically distributed. }
\end{aligned}
$$

If one fixes the premium $P_{t}$ in advance it is important to realize that postulate (2) may now fail with a certain probability. However, one can control this probability of failure (of ruin, as it is usually called). According to a standard formula in risk theory this probability is at most equal to $e^{-k R_{0}}$ where $k>0$ is such that $e^{k(P-C)}=E\left[e^{k S}\right]$.

Hence if we want to control the probability of ruin at the level $\psi_{0}$ (say $1 \%$ ) we may choose

$$
k=\frac{\left|\ln \psi_{0}\right|}{R_{0}}
$$

and calculate the net premium $\Pi=P-C$ from the formula

$$
\Pi=\frac{1}{k} \ln E\left[e^{k S}\right]
$$

(The derivation of this formula from the probability of ruin criterion has been found by DE FineTTI (1939) for the discrete case and by Gerber (1974) for the continuous case).

This formula becomes more accessible to interpretation if we approximate the right-hand side by its second-order Taylor polynomial (with the cumulants as coefficients). 


$$
\begin{aligned}
\Pi & =E[S]+\frac{k}{2} \operatorname{Var}[S] \\
& =E[S]+\frac{\left|\ln \psi_{0}\right|}{2 R_{0}} \operatorname{Var}[S]
\end{aligned}
$$

\subsection{Premium Calculation Principles}

Observe that from our postulate (2) we have arrived in a very natural way at a premium calculation principle

$$
\mathscr{H}: S \rightarrow \Pi
$$

assigning to the yearly total of claims $S$ a yearly total of net premiums $\Pi$.

Our derivation has produced

$$
\text { the exponential principle: } \Pi=\frac{1}{k} \ln E\left[e^{k s}\right]
$$

or approximately

$$
\text { the variance principle: } \quad \Pi=E[S]+\underbrace{\frac{k}{2} \operatorname{Var}[S]}_{\begin{array}{c}
\text { This component is called } \\
\text { the security loading }
\end{array}}
$$

both with $k=\left|\ln \psi_{0}\right| / R_{0}$.

As both of these principles are additive (Definition: $\Pi$ is additive if $\Pi(S+T)=$ $\Pi(S)+\Pi(T)$ for $S, T$ independent) we may apply the formulae (4) and (5) also to individual risks covered by the insurance operation. In doing so we automatically achieve equilibrium in the whole portfolio.

3.4. The Standard Deviation Principle, an Example of a non-additive Premium Calculation Principle.

Formula (5) has lead us to the following rule

$$
\Pi=E[S]+\frac{\left|\ln \psi_{0}\right|}{2 R_{0}} \operatorname{Var}[S]
$$

The operative capital $R_{0}$ is still free in this formula and we may try to optimise its choice.

Up till here we have tacitly disregarded dividends. Let us now consider explicitly dividends to the investor who has provided the initial equity=operative capital $R_{0}$. Assume that we require a total yield of $i R_{0}$ on this operative capital for dividends. 
OBSERVE. $i R_{0}$ is that part of dividends which we want to achieve and which is not tacitly included in the costs. So one might choose the rate of required yield with due respect to the silently included dividend payments under the title of costs.

With this understanding the insurer wants a total premium income (net of costs but including required dividends).

$$
\Pi^{+}=E[S]+\frac{\left|\ln \psi_{0}\right|}{2 R_{0}} \operatorname{Var}[S]+i R_{0}
$$

OBSERVE. $\Pi^{+}=\Pi+i R_{0}$ (net premium + required dividends). This total is the more competitive and hence the more likely to be obtained, the lower it is. The optimum choice of $R_{0}$ is hence the one which renders (6) minimum.

One easily finds that the optimal choice is

$$
R_{0}=\sqrt{\frac{\left|\ln \psi_{0}\right|}{2 i}} \sigma[S], \quad \sigma[S]=\sqrt{\operatorname{Var}[S]} .
$$

The total premium income to the insurer then amounts to

$$
\Pi^{+}=E[S]+\sqrt{2 i\left|\ln \psi_{0}\right|} \sigma[S] .
$$

OBSERVE (a) In the optimum the security loading and the return on initial investment are equal

$$
\begin{aligned}
\frac{\left|\ln \psi_{0}\right|}{2 R_{0}} \operatorname{Var}[S] & =\sqrt{\frac{i\left|\ln \psi_{0}\right|}{2}} \sigma[S] \\
i R_{0} & =\sqrt{\frac{i\left|\ln \psi_{0}\right|}{2}} \sigma[S]
\end{aligned}
$$

(b) $R_{0}$ decreases with increasing $i$ but dividend income $i R_{0}$ is increasing with i.

Formula (8) is the standard deviation principle, which is no longer additive. It hence does not make sense to use Formula (8) for the individual risk. (We would lose control of equilibrium.)

One should rather proceed as follows:

(a) Based on total $S$ calculate

$$
R_{0}=\sqrt{\frac{\left|\ln \psi_{0}\right|}{2 i}} \sigma[S] \quad(\text { formula } 7)
$$

(b) Then use for (independent) individual risks $S_{\text {ind }}$ the variance principle

$$
\Pi_{\text {ind }}^{+}=E\left[S_{\text {ind }}\right]+\frac{\left|\ln \psi_{0}\right|}{R_{0}} \operatorname{Var}\left[S_{\text {ind }}\right] .
$$

(Observe the factor 2 in the loading!) 
One easily checks that the total adds up to

$$
\begin{aligned}
\Pi^{+} & =E[S]+\frac{\left|\ln \psi_{0}\right|}{R_{0}} \operatorname{Var}[S] \\
& =E[S]+\sqrt{2 i\left|\ln \psi_{0}\right|} \sigma[S]
\end{aligned}
$$

which is the correct total according to formula (8)!

Of course there is no absolute necessity to proceed in this way. One however can find different arguments to justify the above procedure as a fair splitting of loadings among individual risks.

Here is one argument for fairness: At each level $R_{0}$ the variance principle applied to individual risks produces a stable portfolio (with probability of ruin $\psi_{0}$ ). It seems then fair to split return on investment $\boldsymbol{R}_{0}$ in the same proportion as the security loadings of the premium.

We shall come back to this question of fairness.

\subsection{An Example}

Assume that our portfolio consists of

$$
5 \text { risks of type A with density } f_{A}(x)=0.2 e^{-0.2 x}(x \geqslant 0)
$$

(exponential distribution with mean 5)

\begin{tabular}{|c|c|c|c|c|c|c|}
\hline & \multirow[b]{2}{*}{$\begin{array}{c}\text { No. of } \\
\text { Risks }\end{array}$} & \multirow[b]{2}{*}{ Mean } & \multirow[b]{2}{*}{ Variance } & \multicolumn{2}{|c|}{$\Pi$} & \multirow[b]{2}{*}{$\begin{array}{l}\text { Stand. Dev. } \\
\text { Principle }\end{array}$} \\
\hline & & & & $\begin{array}{l}\text { Exponential } \\
\text { Principle }\end{array}$ & $\begin{array}{l}\text { Variance } \\
\text { Principle }\end{array}$ & \\
\hline type A & 5 & 5 & 25 & $\frac{1}{k} \ln \left(\frac{0.2}{0.2-k}\right)$ & $5+\frac{k}{2} 25$ & \\
\hline type B & 20 & 1 & 1 & $\frac{1}{k} \ln \left(\frac{1}{1-k}\right)$ & $1+\frac{k}{2} \cdot 1$ & \\
\hline portfolio & & 45 & 145 & & & $45+\sqrt{2 i\left|\ln \psi_{0}\right|} 12.04$ \\
\hline
\end{tabular}

20 risks of type $B$ with density $f_{B}(x)=e^{-x}(e \geqslant 0)$

(exponential distribution with mean 1)

Choose $\psi_{0}=1 \%\left(\left|\ln \psi_{0}\right|=4.6052\right)$.

Then we have for

\begin{tabular}{lccccc}
\hline & $\begin{array}{c}\text { Portfolio } \\
\text { Premium }\end{array}$ & $R_{0}=\sqrt{\frac{\ln \psi_{0}}{2 i} \sigma[S]}$ & Optimum $k$ & $\overbrace{\begin{array}{c}\text { Premium } \\
\text { for Risk } A\end{array}}^{\Pi^{+}}$ & $\begin{array}{c}\text { Premium } \\
\text { for Risk } B\end{array}$ \\
\hline$i=2 \%$ & 50.17 & 129.19 & 0.0356 & 5.89 & 1.0356 \\
$i=5 \%$ & 53.17 & 81.71 & 0.0564 & 6.41 & 1.0564 \\
$i=10 \%$ & 56.55 & 57.77 & 0.0797 & 6.99 & 1.0797 \\
\hline
\end{tabular}


It is easily checked that $20 \times$ premium for risk $B+5 \times$ premium for risk $A$ equals the portfolio premium.

ObSERVE. (1) The higher the return on investment you want the lower this investment must be chosen.

(2) The security loading is by far not proportional to the mean value of the risk. In percent of mean value the loading for risk $A$ is 5 times the loading for risk $B$.

(3) You may check that the exponential principle (with parameter $2 k$ ) leads to premiums which are not drastically different from those obtained by the variance principle.

e.g. $\quad i=2 \%, \quad$ risk $A: 6.18, \quad$ risk $B: 1.037$,

\section{GENERAL PHILOSOPHY}

It should be noted that in the preceding we have calculated from top down.

More explicitly:

(1) We have first considered the total of all risks from a given portfolio (or from a whole insurance company). For this total we have

(i) formulated a stability criterion (e.g., probability of ruin criterion),

(ii) imposed certain conditions regarding yield of invested capital.

(2) The goals set out in (1) have then led us to find a total premium to be charged for the whole portfolio.

(3) In a final step we have then argued how this total should be split in a fair way among all the individual risks.

Our analysis has produced the following pragmatic solution

(a) Fix total premium according to standard deviation principle

$$
\Pi^{+}=E[S]+\sqrt{2 i\left|\ln \psi_{0}\right|} \sigma[S] .
$$

(b) Split the total according to the variance principle (or if you prefer, the exponential principle)

$$
\Pi_{\mathrm{ind}}^{+}=E\left[S_{\mathrm{ind}}\right]+\frac{\left|\ln \psi_{0}\right|}{R_{0}} \operatorname{Var}\left[S_{\mathrm{ind}}\right]
$$

with

$$
R_{0}=\sqrt{\frac{\left|\ln \psi_{0}\right|}{2 i}} \sigma[S]
$$

(c) It is remarkable that our analysis has also produced a theory for choosing an optimal value of invested initial capital. Note that the latter should be proportional to the standard deviation of total claims. 
(d) Note that our analysis also provides an answer to the question about "price for capacity". This price is to be understood as the required yield on the initial investment $\boldsymbol{R}_{\mathbf{0}}$. With the optimum choice of $\boldsymbol{R}_{0}$ this amounts to half of the loading, the other half being used for building up the equity and therewith the protection against risk fluctuations.

This analysis was of course based on a rather simple mode of reality. It is clear that one could work with more intricate model assumptions. Nevertheless the basic philosophy as underlying our analysis is fundamental and should be used for any premium calculation problem.

Unfortunately one still finds actuarial work on premium calculation which takes the contrary viewpoint, namely from bottom up. By this I mean any way of proceeding where one

(i) concentrates on the definition of the correct premium for the individual risk,

(ii) rather neglects to check whether total premium obtained by summation of individual premiums satisfies some reasonable stability criterion.

From the preceding analysis you should hopefully have learned that this bottom up philosophy is unreasonable. Premiums of individual risks are not exclusively determined by this individual risk but (at least in the choice of values of certain parameters) do depend on the portfolio to which they belong.

\section{MARKET PREMIUMS}

So far we have been speaking about permiums for an individual company. The practitioner might call the premium concept so far discussed the technical premium, which is some sort of internal yardstick to find out whether market premiums are in principle acceptable to the individual insurance company or not. Actuaries are mostly only concerned with this technical premium. Nevertheless it might be useful to study some models of market behaviour to find out what tendencies one might expect from market conditions. Such a simple model is studied in BuehlmanN (1980). Let me explain the basic result. The effect of a competitive market can be viewed as if the basic probabilities were changed. If state $\omega$ has objective probability $p(\omega)$ the market premium will be obtained from the modified probabilities

$$
\frac{e^{\alpha Z(\omega)} p(\omega)}{\sum_{\omega^{\prime}} e^{\alpha Z\left(\omega^{\prime}\right)} p\left(\omega^{\prime}\right)}
$$

where $Z(\omega)$ stands for the total of claims to the market if state $\omega$ happens and where $\alpha$ is a measure of total risk aversion. From this analysis one might conclude that market premiums are more likely to meet the standards of technical premiums if

(i) insurances is on states which cause a high total of claims to the market, and/or

(ii) risk aversion for the states insured is rather high. 


\section{VARIABLE PREMIUMS}

Except for the case of the pool where the total of net premiums follows exactly the claims we have-in this paper-treated premiums as fixed quantities, known in advance before entering the insurance contract.

Of course, there are intermediate ways: The extreme cases of flat premium on one side and totally claims dependent premiums on the other side may be combined. This is essentially the philosophy in experience rating and in particular in credibility theory. I shall not treat this very important field of rating techniques in this publication. But permit me one remark:

Credibility is another good example of a from top down approach to premium calculation. It essentially also solves the problem of fair splitting of total premium among the individual risks.

\section{GROWING BUSINESS}

In paragraph (2) we have mentioned that only in stationary situations it makes sense to finance the insurance operation only at the beginning through the initial investment $R_{0}$.

With growing business one must "adjust" $R_{0}$ according to the growth of standard deviation $\sigma[S]$.

To illustrate this assume in our example 3.5 that the portfolio has doubled (10 risks of type $A, 40$ risks of type $B$ ). Then the respective table has to be modified as follows

\begin{tabular}{lrcccc}
\hline & $\begin{array}{c}\text { Portfolio } \\
\text { Premium }\end{array}$ & Optimum $R$ & Optimum $k$ & $\begin{array}{c}\text { Premium } \\
\text { for Risk } A\end{array}$ & $\begin{array}{c}\text { Premium } \\
\text { for Risk } B\end{array}$ \\
\hline$i=2 \%$ & 97.31 & 182.70 & 0.0252 & 5.630 & 1.0252 \\
$i=5 \%$ & 101.55 & 115.56 & 0.0399 & 5.998 & 1.0399 \\
$i=10 \%$ & 106.33 & 81.70 & 0.0564 & 6.409 & 1.0564 \\
\hline
\end{tabular}

Observe that.

$\begin{array}{lr}\begin{array}{l}\text { loading of portfolio premium increased by } \sqrt{2}, \\ \text { optimum } R\end{array} & \text { increased by } \sqrt{2}, \\ \text { optimum } k & \text { decreased by } \frac{1}{\sqrt{2}}, \\ \text { loading of ind. premium } & \text { decreased by } \frac{1}{\sqrt{2}} .\end{array}$

These observations could of course be directly made from the formulae (5), (7), (8). 
The question arises how one has to interpret the new financial requirements. (For the clear analysis of the problem assume that doubling of the business occurs instantaneously at time $t_{0}$.)

One might argue in two different ways (at the $5 \%$ interest level e.g.).

I At time $t_{0}$ optimum $R=115.56$ is needed At time 0 optimum $R_{0}=81.71$ was invested

Remainder to be invested now 33.85

II At time $t_{0}$ optimum $R=115.56$ is needed

At time $t_{0} R_{0}$ has grown

$$
\text { to, say }=105.30
$$

So only

10.26 additional investment is needed

In my view argument $\mathrm{I}$ is the correct one. Argument II defines a hidden dividend payment which changes the probability of ruin. If we want to be consistent with our criterion of stability II should be abolished. Nevertheless it is often encountered in practical applications with the idea that one starts afresh at time $t_{0}$. It is worthwhile to note that already DE FINETTI (1957) has criticised this argument in his famous paper to the International Congress of Actuaries. I fully share his criticism.

8. ANOTHER FORMULATION OF EQUILIBRIUM AND ITS CONSEQUENCE ON BOTH PORTOLIO AND INDIVIDUAL PREMIUM

\subsection{Motivation}

Remember that we started from the basic recursive relation for surplus

$$
R_{t}=\left(1+i_{t}\right) R_{t-1}+\Pi_{t}-S_{t} ; \quad t=1,2,3, \ldots
$$

We then formulated equilibrium (stable functioning of insurance) by the postulate

$$
R_{t} \geqslant 0 \text { for all } t=1,2,3, \ldots
$$

In the case of prospectively calculated premiums we did assume (as we said, partially for didactical reasons)

$$
\left.\begin{array}{c}
i_{t}=0 \\
\Pi_{t}=\Pi
\end{array}\right\} \quad \text { for all } t=1,2,3, \ldots
$$

$S_{1}, S_{2}, \ldots, S_{t}, \ldots$ independent and identically distributed. These assumptions have allowed us a rather straight forward derivation of the required premiums. One might, however, object that we have oversimplified reality. In particular one might object that we have completely ignored interest on surplus. In this section I would like to show that interest on surplus changes-of course-some quantitative aspects of premium calculation. This is not surprising. The message of this 
section is, however, that the basic formulae for the portfolio premiums (and consequently also individual premiums) remain the same whether we include interest on surplus or not.

\subsection{New Formulation of Equilibrium}

Let us now assume

$$
\left.\begin{array}{r}
i_{t}=i \\
\Pi_{t}=\Pi
\end{array}\right\} \quad \text { for all } t
$$

$S_{1}, S_{2}, \ldots, S_{t}, \ldots$ independent and identically distributed (mean $\mu$, standard deviation $\sigma$ ) and let us now express equilibrium by

$$
R_{t} \geqslant-\frac{\Pi}{i} \text { for all } t=1,2, \ldots
$$

Observe that we have moved from a very conservative formulation (no interest on surplus) to a very liberal one. The liberal formulation is not only characterized by the inclusion of interest but also by the relaxation of the solvency standard (postulate (9)). Observe that (9) allows surplus also to become negative up to the point where premiums still suffice to finance the negative interest created by the surplus deficit.

This relaxation is of course very generous. We go here from one extreme (no interest on reserves) to the other. But by analysing the two extremes (and-as we shall see-by finding out that consequences on premiums required are not that drastic) we can conclude for practical applications that premiums ought to lie between the values generated by these two boundary cases.

\subsection{Calculation of Portfolio Premiums}

(Ideas in this part are heavily borrowed from GERBER (1971).)

Introduce first the present value $B_{t}$ of surplus, defined as follows

$$
B_{t}=(1+i)^{-t} R_{t}=v^{t} R_{t}
$$

Observe that

$$
B_{\infty}=R_{0}+\sum_{j=1}^{\infty} v^{j}\left(\Pi-S_{j}\right)
$$

is almost surely finite if $E\left[S_{j}\right]$ and $\operatorname{Var}\left[S_{j}\right]$ are finite.

The following lemma is essential

LEMMA
(a) $R_{t} \geqslant-\frac{\Pi}{i}$ for all $t \Rightarrow B_{\infty} \geqslant 0$,
(b) $R_{t}<-\frac{\Pi}{i}$ for one $t \Rightarrow B_{\infty}<0$. 
Proof. Use the fact that:

$$
R_{t_{0}}=-\frac{\Pi}{i}-\delta_{t_{0}} \Rightarrow R_{\mathrm{t}} \leqslant-\frac{\Pi}{i}-\delta_{t_{0}}(1+i)^{t-t_{0}} \quad \text { for all } t \geqslant t_{0} .
$$

Hence we can formulate equilibrium by

$$
B_{\infty} \geqslant 0 \text {. }
$$

Rewriting condition (12) with the use of (11) we have

$$
\sum_{j=1}^{\infty} v^{j} S_{j} \leqslant R_{0}+\frac{\Pi}{i} .
$$

According to the Discounted Central Limit Theorem the left-hand side of (13) has approximately a normal distribution with mean $\mu / i$ standard deviation $\sigma / \sqrt{2 i+i^{2}}$.

Let $\delta_{\varepsilon}$ be the argument for which we have probability $\varepsilon$ in the right tail of the standard normal distribution.

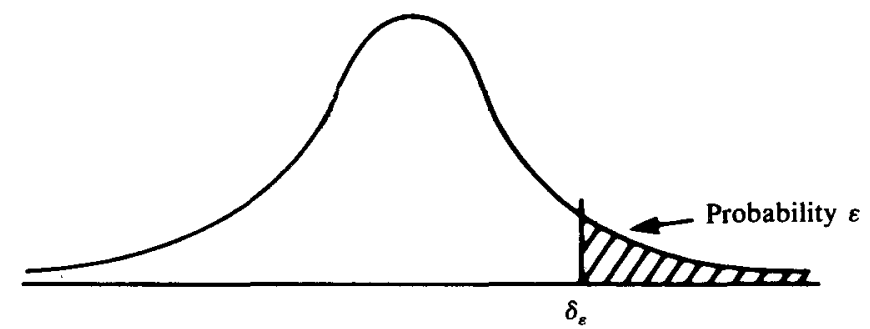

Equilibrium is then guaranteed with probability $\varepsilon$ if

$$
\frac{R_{0}+\Pi / i-\mu / i}{\sigma / \sqrt{2 i+i^{2}}}=\delta_{\varepsilon} \text {. }
$$

Hence

$$
\begin{gathered}
\Pi=\mu+\delta_{\varepsilon} \sigma \frac{i}{\sqrt{2 i+i^{2}}}-i R_{0} \\
\sim \mu+\frac{\delta_{\varepsilon}}{\sqrt{2}} \sigma \sqrt{i}-i R_{0} .
\end{gathered}
$$

If we allow explicitely for dividends in our premium calculation (charging an additional payment $i R_{0}$ for dividends) we arrive at

$$
\Pi^{+}=\mu+\frac{\delta_{\varepsilon}}{\sqrt{2}} \sigma \sqrt{i}
$$


Let us stop here and pause for a moment. Several observations are important to be made now:

(1) Miraculously formula (15) and formula (8) have exactly the same structure. The only difference is that safety is expressed in some other measures

Formula 8: $\quad \sqrt{\left|2 \ln \psi_{0}\right|}$

Formula 15: $\frac{\delta_{\varepsilon}}{\sqrt{2}}$.

The following table allows a comparison of the values obtained by these different measures.

\begin{tabular}{ccc}
\hline \hline Probability of Ruin & $\delta_{e} / \sqrt{2}$ & $\sqrt{\left|2 \ln \psi_{0}\right|}$ \\
\hline $5 \%$ & 1.16 & 2.45 \\
$1 \%$ & 1.64 & 3.03 \\
$1 \%$ & 2.19 & 3.72 \\
\hline
\end{tabular}

It is most remarkable that the two models lead to parameter values which are not drastically different!

(2) Observe that our new formulation has not provided us with a theory of how to optimize $R_{0}$. In a way all $R_{0}$ are equally reasonable. Of course this would be changed if we were not using the same interest rate to finance dividends and to augment technical surplus.

\section{ACKNOWLEDGMENT}

I should like to thank particularly one of the referees who had a real thorough look at the paper and has made valuable suggestions.

\section{BIBLIOGRAPHY}

Buehlmann, H. (1980) An Economic Premium Principle. ASTIN Bulletin 11, 52-60.

DE Finetti, B. (1939). La Teoria del Rischio e il Problema della Rovina dei Giocatori. Giornale dell' Istituto Italiano degli Astuari X, 1-2.

DE FINETTI, B. (1957) Su un'Impostazione Alternativa della Teoria Collettiva del Rischio. Trans. $X V . I C A$ 2, 433-443.

Gerber, H. U. (1971). Der Einfluss von Zins auf die Ruinwahrscheinlichkeit. Mitteilungen der Vereinigung Schweizerischer Versicherungsmathematiker 71, 1.

Gerber, H. U. (1974). On Additive Premium Calculation Principles. ASTIN Bulletin VII, 3. 